\title{
Decreased circulating dihomo-gamma- linolenic acid levels are associated with total mortality in patients with acute cardiovascular disease and acute decompensated heart failure
}

Shohei Ouchi, Tetsuro Miyazaki, Kazunori Shimada, Yurina Sugita, Megumi Shimizu, Azusa Murata, Takao Kato, Tatsuro Aikawa, Shoko Suda, Tomoyuki Shiozawa, Masaru Hiki, Shuhei Takahashi, Takatoshi Kasai,

Katsumi Miyauchi and Hiroyuki Daida

\begin{abstract}
Background: Polyunsaturated fatty acids (PUFAs) have important roles in the pathogenesis of cardiovascular diseases. However, the clinical significance of omega-6 PUFAs in acute cardiovascular disease remains unknown.

Methods: We enrolled 417 consecutive patients with acute cardiovascular disease admitted to the cardiac intensive care unit at Juntendo University Hospital between April 2012 and October 2013. We investigated the association between serum PUFA levels and long-term mortality. Blood samples were collected after an overnight fast, within $24 \mathrm{~h}$ of admission. We excluded patients who received eicosapentaenoic acid therapy and those with malignancy, endstage kidney disease, chronic hepatic disease, and connective tissue disease.

Results: Overall, 306 patients (mean age: $66.4 \pm 15.0$ years) were analysed. During the follow-up period of $2.4 \pm 1.2$ years, 50 patients (16.3\%) died. The dihomo-gamma-linolenic acid (DGLA) levels, arachidonic acid (AA) levels, and DGLAVAA ratio were significantly lower in the nonsurvivor group than in the survivor group (DGLA: $23.2 \pm 9.8 \mathrm{vs} .31 .5 \pm 12.0 \mathrm{\mu g} / \mathrm{ml}$, AA: $151.1 \pm 41.6$ vs. $173.3 \pm 51.6 \mu \mathrm{g} / \mathrm{ml}$, and DGLAVAA: $0.16 \pm 0.05$ vs. $0.19 \pm 0.06$, all $p<0.01$ ). Kaplan-Meier curves showed that survival rates were significantly higher in the higher DGLA, AA, and DGLA/AA groups than in their lower counterparts (DGLA and AA; $p<0.01$, DGLA/AA; $p=0.01$ ), although omega-3 PUFAs were not associated with prognosis. Furthermore, in patients with acute decompensated heart failure (ADHF), survival rates were significantly higher in the higher DGLA, $A A$, and DGLA/AA groups than in their lower counterparts (DGLA and $A A ; p<0.01, D G L A / A A ; p=0.04$ ). However, among patients with acute coronary syndrome, none of the PUFA levels were associated with prognosis. Among patients with $A D H F$, after controlling for confounding variables, DGLA and DGLA/AA were associated with long-term mortality [DGLA: hazard ratio (HR), 0.94; 95\% confidence interval (Cl), 0.88-0.99; $p=0.01$ and DGLA/AA: HR, 0.87; 95\% Cl, $0.77-0.97 ; p<0.01]$, whereas AA was not associated with prognosis.
\end{abstract}

Conclusion: Low omega-6 PUFA levels, particularly DGLA, and a low DGLA/AA ratio predict long-term mortality in patients with acute cardiovascular disease and ADHF.

Trial registration: UMIN-CTR; UMIN000007555.

Keywords: Polyunsaturated fatty acids, Dihomo-gamma-linolenic acid, Arachidonic acid, Omega-6, Inflammation, Nutrition

\footnotetext{
* Correspondence: tetsuro@juntendo.ac.jp

Department of Cardiovascular Medicine, Juntendo University School of

Medicine, 2-1-1 Hongo Bunkyo-ku, Tokyo 113-8421, Japan
} 


\section{Background}

Polyunsaturated fatty acids (PUFAs) play structural and functional roles as membrane components and precursors for physiologically active substances involved in inflammation [1]. PUFAs are characterized by the presence of at least two carbon-carbon double bonds. Omega-3 PUFAs [eicosapentaenoic acid (EPA) and docosahexaenoic acid (DHA)] have the first double bond at the third carbon from the methyl terminus, and omega-6 PUFAs [arachidonic acid (AA) and dihomo-gamma-linolenic acid (DGLA)] have the first double bond at the sixth carbon from the methyl terminus [2, 3]. Fish oils are rich in omega-3 PUFAs, whereas farm animals and sunflower, safflower, and corn oils are rich in omega-6 PUFAs [4, 5]. A previous study reported that in Japanese individuals, the levels of EPA, DHA, EPA/AA, and other omega-3/ omega-6 PUFAs in serum and red blood cells gradually increased with age, owing to the westernization of lifestyles in young people including the increased availability of high-fat foods [6].

PUFAs, particularly omega-3 PUFAs, have several important roles in the prevention of cardiovascular diseases and have anti-inflammatory and antiatherogenic effects [7-14]. Previous studies have reported that treatment with omega-3 PUFAs increases the left ventricular ejection fraction in patients with chronic heart failure [5, 15-17]. However, it is unclear whether omega-6 PUFAs are beneficial or harmful with regard to the prevention of cardiovascular diseases [18-22]. Moreover, the clinical significance of PUFA metabolism, including that of omega-6 PUFAs, in acute cardiovascular diseases remains unknown. Therefore, we investigated the association between serum PUFA levels and prognosis in patients with acute cardiovascular disease.

\section{Methods}

\section{Patients}

The present study was a part of an ongoing cohort study of biomarkers in patients admitted to the cardiac intensive care unit (UMIN-CTR; UMIN000007555), in which the PUFA hypothesis was generated retrospectively; however, data were collected systematically and prospectively. We enrolled 417 consecutive patients with cardiovascular diseases admitted to the cardiac intensive care unit at Juntendo University Hospital between April 2012 and October 2013. We excluded patients who received EPA therapy and those with malignancy, endstage kidney disease [defined as an estimated glomerular filtration rate (eGFR) $\left.<15 \mathrm{ml} / \mathrm{min} / 1.73 \mathrm{~m}^{2}\right]$, chronic hepatic disease, and connective tissue disease (Fig. 1). The primary endpoint was all-cause death, and we followed up with the patients until December 2015.

Overall, 306 patients were analysed in this study. The mean patient age was $66.4 \pm 15.0$ years. Of the 306 patients, 209 (68.3\%) were male. All patients were Japanese. Juntendo University Hospital is located in Tokyo, the capital of Japan, and none of the patients were employed in a primary industry. Based on socioeconomic status, the patients included 145 retirees or housewives (47.4\%), 130 white-collar workers (42.5\%), 6 physical laborers $(2.0 \%)$, and 5 who received public assistance (1.6\%). The occupations of the other 20 patients $(6.5 \%)$ were unclear from the medical records. The mean follow-up duration was $2.4 \pm 1.2$ years, and the maximum follow-up duration was 3.7 years.

Acute decompensated heart failure (ADHF) was defined according to the diagnostic criteria of the Framingham study [23]. Acute coronary syndrome (ACS) was defined as having an unstable angina pectoris, a non-ST elevation, or a ST elevation myocardial infarction. Diabetes mellitus

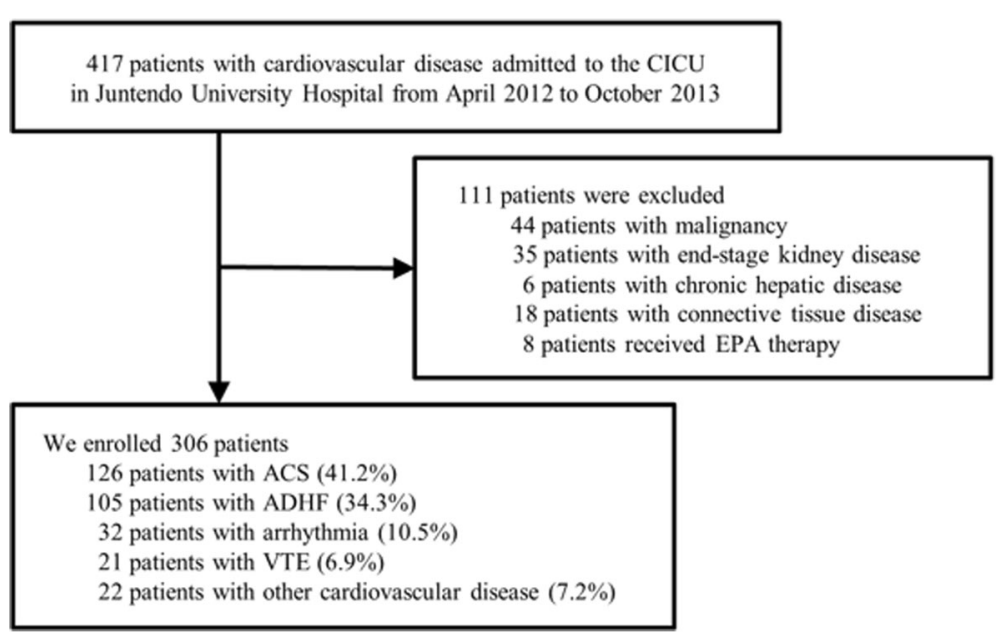

Fig. 1 Flowchart of the study population 
was defined as having a previous diagnosis of diabetes mellitus in the medical records, a hemoglobin A1c (national glycohemoglobin standardization program calculation) level $\geq 6.5 \%$, or treatment with oral antidiabetic agents or insulin. Dyslipidemia was defined as having a previous diagnosis of dyslipidemia in the medical records, abnormal lipid profiles [i.e., triglyceride (TG) level $\geq 150 \mathrm{mg} / \mathrm{dl}$, lowdensity lipoprotein cholesterol (LDL-C) level $\geq 140 \mathrm{mg} / \mathrm{dl}$, or high-density lipoprotein cholesterol (HDL-C) level $\leq 40 \mathrm{mg} / \mathrm{dl}]$, or treatment with antidyslipidemic agents. Hypertension was defined as having a previous diagnosis of hypertension, which was defined as having a systolic blood pressure of $\geq 140 \mathrm{mmHg}$ and/or diastolic blood pressure of $\geq 90 \mathrm{mmHg}$ [24] in the medical records or treatment with antihypertensive agents. All subjects provided informed consent, and the study was approved by the Ethical Committee of Juntendo University Hospital.

\section{Blood sampling}

Whole blood samples were collected after an overnight fast, within $24 \mathrm{~h}$ of admission, and were analysed using standardized methods. Tubes for measuring PUFAs contained heparin sodium, those for measuring HbA1c levels contained heparin sodium, ethylenediaminetetraacetic acid disodium salt and sodium fluoride, those for measuring brain natriuretic peptide (BNP) levels contained ethylenediaminetetraacetic acid dipotassium salt, and those for measuring total cholesterol, TG, HDL-C, creatinine, total protein, and albumin contained a fast-acting coagulant. The LDL-C level was calculated using the Friedewald formula. eGFR was calculated based on the Japanese equation that uses serum creatinine level, age, and gender as follows: eGFR ( $\mathrm{mL} / \mathrm{min} /$ $\left.1.73 \mathrm{~m}^{2}\right)=194 \times$ creatinine $^{-1.094} \times$ age $^{-0.287}($ for females $=\times$ 0.739) [25]. Serum concentrations of EPA, DHA, DGLA, and AA were measured using standard laboratory protocols by SRL Inc. (Tokyo, Japan).

\section{Statistical analyses}

Continuous variables were expressed as means with standard deviations, and categorical variables were expressed as counts and percentages. Comparisons of continuous variables were performed using the Student's $t$-test or Mann-Whitney $U$-test. Categorical variables were analysed using the chi-square test or Fisher's exact probability test. Correlations between two variables were determined using simple linear regression analysis. The patients were divided into survivor or nonsurvivor groups based on their survival. Unadjusted cumulative event rates for the primary endpoint were estimated using the Kaplan-Meier method and were compared between groups using the log-rank test. We defined cutoffs using the median PUFA levels. We analysed the long-term mortality of patients with ADHF and those with ACS. Among patients with ADHF, univariate and multivariate Cox regression analyses were performed to identify predictors of the primary endpoint. The hazard ratios (HRs) and 95\% confidence intervals (CIs) were also calculated. Age, sex, body mass index (BMI), diabetes mellitus, dyslipidemia, hypertension, renal function (eGFR), atrial fibrillation, and cardiac function (left ventricular ejection fraction) were included in the multivariate analyses. JMP12 (for Windows, SAS Institute, Cary, NC) was used for the statistical analysis, and $p$-values $<0.05$ were considered statistically significant.

\section{Results}

The survivor group included 256 patients (83.7\%), and the nonsurvivor group included 50 patients (16.3\%). Age and prevalence of atrial fibrillation were significantly higher and BMI, systolic blood pressure, diastolic blood pressure, ejection fraction, and prevalence of dyslipidemia were significantly lower in the nonsurvivor group than in the survivor group. Total cholesterol, LDL-C, TG, and albumin levels were significantly lower, BNP levels were significantly higher, and impaired renal function was more common in the nonsurvivor group than in the survivor group. The administration of diuretics, anticoagulants, and inotropic agents was more common in the nonsurvivor group than in the survivor group (Table 1).

As shown in Fig. 2, the DGLA and AA levels were significantly lower in the nonsurvivor group than in the survivor group (DGLA: $23.2 \pm 9.8$ vs. $31.5 \pm 12.0 \mu \mathrm{g} / \mathrm{ml}$ and AA: $151.1 \pm 41.6$ vs. $173.3 \pm 51.6 \mu \mathrm{g} / \mathrm{ml}$, all $p<0.01)$, although the EPA and DHA levels did not differ between the groups. The DGLA/AA ratio, which indicates the conversion rate of DGLA to AA, was significantly lower in the nonsurvivor group than in the survivor group $(0.16 \pm 0.05$ vs. $0.19 \pm 0.06, p<0.01)$.

In this study population, DGLA was negatively related to age, heart rate, and BNP level and positively related to BMI, systolic blood pressure, diastolic blood pressure, ejection fraction, and eGFR as well as to total cholesterol, LDL-C, HDL-C, TG, total protein, and albumin levels (all $p<0.05$ ). AA was negatively related to age, heart rate, and BNP level and positively related to BMI, systolic blood pressure, diastolic blood pressure, and eGFR as well as to total cholesterol, LDL-C, HDL-C, TG, and albumin levels (all $p<0.05$ ). EPA was negatively related to BNP level and positively related to age, systolic blood pressure, diastolic blood pressure, and ejection fraction as well as to total cholesterol, LDL-C, and HDL-C levels (all $p<0.05$ ). DHA was negatively related to BNP level and positively related to age, systolic blood pressure, diastolic blood pressure, and ejection fraction as well as to total cholesterol, LDL-C, HDL-C, TG, and 
Table 1 Characteristics of the study subjects

\begin{tabular}{|c|c|c|c|}
\hline & Survivor group $(n=256)$ & Nonsurvivor group $(n=50)$ & $p$ \\
\hline Age (years) & $64.7 \pm 15.2$ & $75.3 \pm 10.7$ & $<0.01$ \\
\hline Male (n, \%) & $175(68.4)$ & $34(68.0)$ & NS \\
\hline Body mass index $\left(\mathrm{kg} / \mathrm{m}^{2}\right)$ & $24.2 \pm 4.5$ & $22.1 \pm 3.8$ & $<0.01$ \\
\hline Systolic blood pressure $(\mathrm{mmHg})$ & $134 \pm 27$ & $124 \pm 32$ & 0.03 \\
\hline Diastolic blood pressure (mmHg) & $78 \pm 19$ & $70 \pm 18$ & $<0.01$ \\
\hline Heart rate (/min) & $85 \pm 30$ & $82 \pm 25$ & NS \\
\hline Ejection fraction (\%) & $51.6 \pm 16.7$ & $39.7 \pm 20.1$ & $<0.01$ \\
\hline Diabetes mellitus ( $\mathrm{n}, \%)$ & $86(33.6)$ & $21(42.0)$ & NS \\
\hline Dyslipidemia (n, \%) & $191(74.6)$ & $28(56.0)$ & 0.01 \\
\hline Hypertension (n, \%) & $174(68.0)$ & $34(68.0 \%)$ & NS \\
\hline Atrial fibrillation $(\mathrm{n}, \%)$ & $59(23.1)$ & $22(44.0)$ & $<0.01$ \\
\hline Ischemic heart disease (n, \%) & $141(55.1)$ & $27(54.0)$ & NS \\
\hline \multicolumn{4}{|l|}{ Diagnosis } \\
\hline Acute decompensated heart failure $(n, \%)$ & $71(27.7)$ & $34(68.0)$ & $<0.01$ \\
\hline Acute coronary syndrome $(n, \%)$ & $121(47.3)$ & $8(16.0)$ & $<0.01$ \\
\hline \multicolumn{4}{|l|}{ Laboratory data } \\
\hline Total cholesterol (mg/dL) & $172 \pm 38$ & $153 \pm 27$ & $<0.01$ \\
\hline LDL-cholesterol (mg/dL) & $105 \pm 31$ & $93 \pm 21$ & 0.03 \\
\hline HDL- cholesterol (mg/dL) & $44 \pm 14$ & $42 \pm 12$ & NS \\
\hline Triglycerides (mg/dL) & $116 \pm 72$ & $86 \pm 39$ & 0.01 \\
\hline eGFR $\left(\mathrm{mL} / \mathrm{min} / 1.73 \mathrm{~m}^{2}\right)$ & $71.9 \pm 27.4$ & $45.4 \pm 26.8$ & $<0.01$ \\
\hline Hemoglobin A1c (\%) & $6.2 \pm 1.2$ & $6.1 \pm 0.8$ & NS \\
\hline Total protein (g/dL) & $6.6 \pm 0.6$ & $6.5 \pm 0.6$ & NS \\
\hline Albumin $(\mathrm{g} / \mathrm{dL})$ & $3.8 \pm 0.6$ & $3.4 \pm 0.5$ & $<0.01$ \\
\hline Brain natriuretic peptide $(\mathrm{pg} / \mathrm{mL})$ & $490 \pm 762$ & $1443 \pm 1357$ & $<0.01$ \\
\hline \multicolumn{4}{|l|}{ Medication } \\
\hline Diuretics (n, \%) & $45(17.7)$ & $29(59.2)$ & $<0.01$ \\
\hline Antiplatelets (n, \%) & $95(37.4)$ & $25(51.0)$ & NS \\
\hline Anticoagulants (n, \%) & $39(15.4)$ & $22(44.9)$ & $<0.01$ \\
\hline ACE-I/ARBs $(n, \%)$ & $111(43.7)$ & $29(59.2)$ & NS \\
\hline$\beta$-blockers (n, \%) & $82(32.3)$ & $22(44.9)$ & NS \\
\hline Calcium channel blockers (n, \%) & $94(37.0)$ & $18(36.7)$ & NS \\
\hline Inotropic agents (n, \%) & $3(1.2)$ & $9(18.4)$ & $<0.01$ \\
\hline Statins $(n, \%)$ & $81(31.9)$ & $16(32.7)$ & NS \\
\hline Oral hypoglycemic agents (n, \%) & $45(17.7)$ & $9(18.4)$ & NS \\
\hline Insulin (n, \%) & $15(5.9)$ & $3(6.1)$ & NS \\
\hline
\end{tabular}

Data are presented as mean \pm standard deviation or number (percentage)

LDL-cholesterol = low-density lipoprotein cholesterol, HDL-cholesterol = high-density lipoprotein cholesterol, eGFR = estimated glomerular filtration rate, $A C E-I=$ angiotensin converting enzyme inhibitor, ARB = angiotensin-2 receptor blocker

albumin levels (all $p<0.05$ ). DGLA/AA was negatively related to age and BNP level and positively related to BMI and eGFR as well as to total cholesterol, LDL-C, TG, total protein, and albumin levels (all $p<0.05$ ). EPA/ AA was positively related to age, systolic blood pressure, and diastolic blood pressure (all $p<0.05$ ).
Kaplan-Meier curves were constructed to show the unadjusted event-free rate for all-cause death. We divided the patients into two groups based on the median level of each PUFA (DGLA: $28.5 \mu \mathrm{g} / \mathrm{ml}$, AA: $162.0 \mu \mathrm{g} /$ $\mathrm{ml}$, EPA: $46.1 \mu \mathrm{g} / \mathrm{ml}$, DHA: $123.2 \mu \mathrm{g} / \mathrm{ml}$, DGLA/AA: 0.175, and EPA/AA: 0.29). The event-free survival rates 


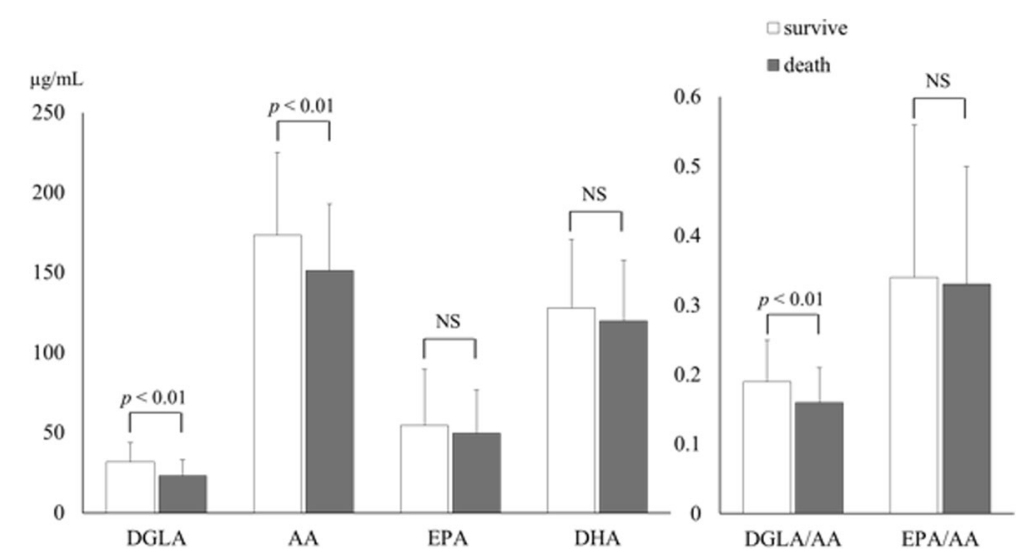

Fig. 2 Comparison of polyunsaturated fatty acid levels between the survivor and nonsurvivor groups. Dihomo-gamma-linolenic acid (DGLA) and arachidonic acid (AA) levels and DGLA/AA ratio were significantly lower in the nonsurvivor group than in the survivor group, whereas eicosapentaenoic acid (EPA) and docosahexaenoic acid (DHA) levels and EPA/AA ratio did not differ between groups

were higher in the higher DGLA, AA, and DGLA/AA groups than in their lower counterparts (DGLA: $p<0.01$, AA: $p<0.01$, and DGLA/AA: $p=0.01$ ), although the EPA and DHA levels and the EPA/AA ratio were not associated with prognosis (Fig. 3).

Furthermore, we investigated the prognosis in patients with ADHF and those with ACS. We divided the patients with ADHF (105 patients) into two groups based on the median level of each PUFA (DGLA: $22.1 \mu \mathrm{g} / \mathrm{ml}$, AA: $147.2 \mu \mathrm{g} / \mathrm{ml}$, EPA: $38.2 \mu \mathrm{g} / \mathrm{ml}$, DHA: $113.3 \mu \mathrm{g} / \mathrm{ml}$,
DGLA/AA: 0.15, and EPA/AA: 0.27). The event-free survival rates were higher in the higher DGLA, AA, and DGLA/AA groups than in their lower counterparts (DGLA: $p<0.01$, AA: $p<0.01$, and DGLA/AA: $p=0.04$; Fig. 4). However, among patients with ACS (129 patients), none of the PUFA levels were associated with prognosis.

Among patients with ADHF, univariate Cox regression analyses showed that age, BMI, dyslipidemia, eGFR, DGLA, AA, DHA, and DGLA/AA, but not EPA and
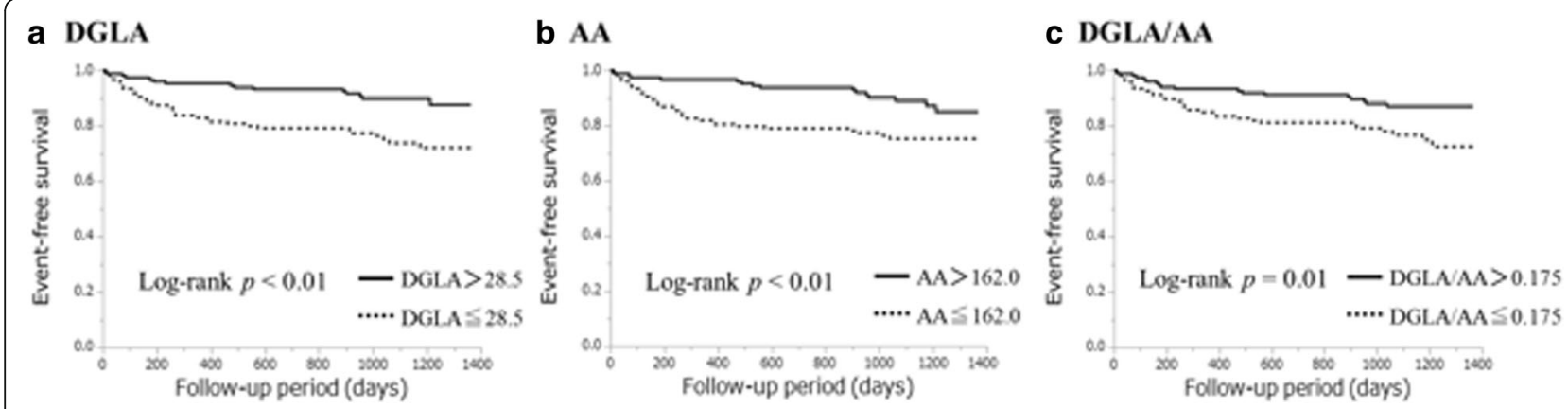

\section{d EPA}
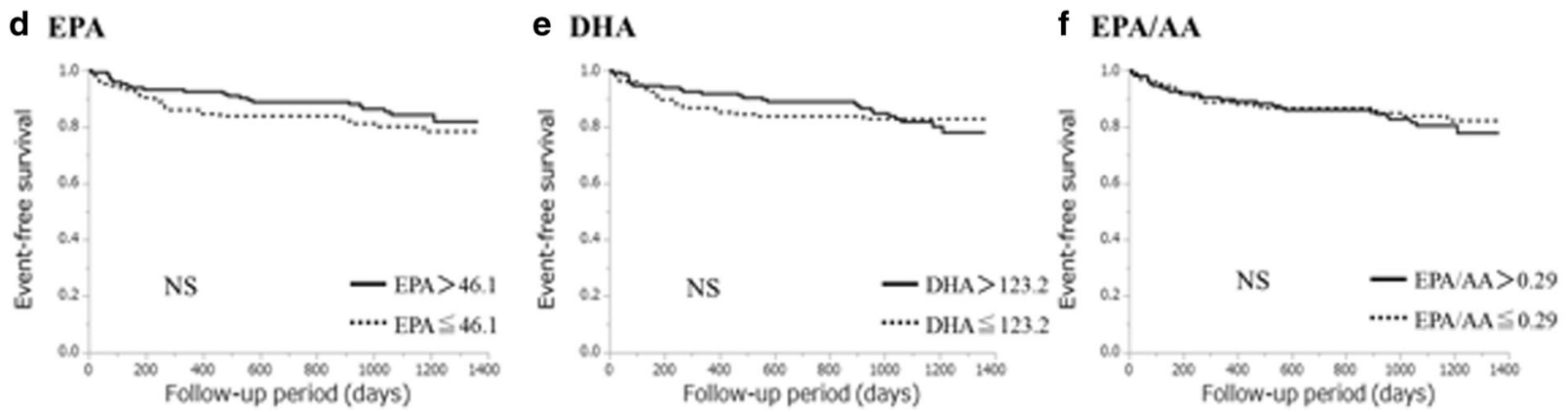

Fig. 3 Event-free survival curves for all-cause death in patients with cardiovascular disease. Unadjusted cumulative event rates for the primary endpoint (all-cause death) were estimated using the Kaplan-Meier method and compared between the groups using the log-rank test. We defined cut-offs as the median levels of polyunsaturated fatty acids [dihomo-gamma-linolenic acid (DGLA), $28.5 \mu \mathrm{g} / \mathrm{ml}$; arachidonic acid (AA), $162.0 \mu \mathrm{g} / \mathrm{ml}$; eicosapentaenoic acid (EPA), $46.1 \mu \mathrm{g} / \mathrm{ml}$; docosahexaenoic acid (DHA), $123.2 \mu \mathrm{g} / \mathrm{ml}$; DGLAVAA, 0.175; EPAAAA, 0.29] 


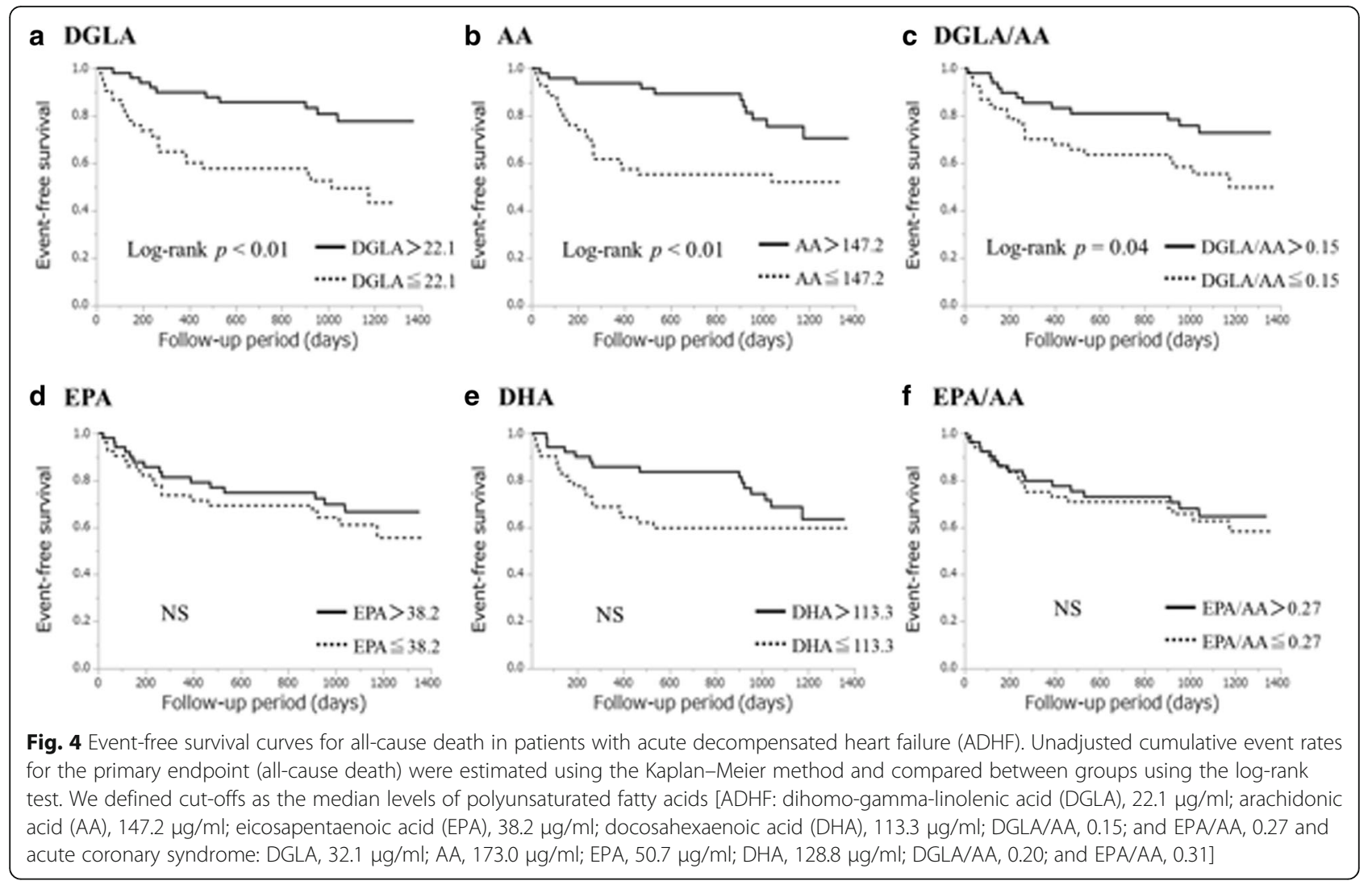

EPA/AA, were associated with long-term mortality (Table 2). After controlling for confounding variables, DGLA and DGLA/AA were associated with long-term mortality (DGLA, $1 \mu \mathrm{g} / \mathrm{ml}$ increase: HR, 0.94; 95\% CI, $0.89-0.99 ; p=0.03$ and DGLA/AA, $0.01 \mu \mathrm{g} / \mathrm{ml}$ increase: HR, 0.87; 95\% CI, 0.78-0.98; $p=0.02$ ), whereas AA, EPA, DHA, and EPA/AA were not associated with longterm mortality (Table 2).

\section{Discussion}

The present study demonstrated that low serum levels of omega-6 PUFAs in patients with acute cardiovascular disease could predict poor long-term prognosis, but omega-3 PUFA levels were not associated with prognosis. Particularly in patients with ADHF, lower serum levels of DGLA and a lower DGLA/AA ratio could predict poor long-term prognosis, whereas in

Table 2 Univariate and multivariate Cox regression analyses for all-cause death

\begin{tabular}{|c|c|c|c|c|c|c|c|c|c|c|c|c|}
\hline & \multicolumn{3}{|c|}{ Univariate } & \multicolumn{3}{|c|}{ Multivariate (AA) } & \multicolumn{3}{|c|}{ Multivariate (DGLA) } & \multicolumn{3}{|c|}{ Multivariate (DGLA/AA) } \\
\hline & $\overline{\mathrm{HR}}$ & $95 \% \mathrm{Cl}$ & $p$ & $\overline{\mathrm{HR}}$ & $95 \% \mathrm{Cl}$ & $p$ & $\overline{\mathrm{HR}}$ & $95 \% \mathrm{Cl}$ & $p$ & $\overline{\mathrm{HR}}$ & $95 \% \mathrm{Cl}$ & $p$ \\
\hline Age, 1 year increase & 1.04 & $1.01-1.07$ & $<0.01$ & 1.02 & $0.98-1.06$ & NS & 1.01 & $0.97-1.05$ & NS & 1.00 & $0.97-1.04$ & NS \\
\hline Male & 1.45 & $0.73-3.02$ & NS & 1.25 & $0.61-2.71$ & NS & 1.41 & $0.67-3.12$ & NS & 1.43 & $0.68-3.14$ & NS \\
\hline Body mass index, $1 \mathrm{~kg} / \mathrm{m}^{2}$ increase & 0.90 & $0.83-0.97$ & $<0.01$ & 0.95 & $0.87-1.04$ & NS & 0.97 & $0.88-1.06$ & NS & 0.95 & $0.87-1.04$ & NS \\
\hline Diabetes mellitus & 1.24 & $0.63-2.44$ & NS & 1.29 & $0.56-3.05$ & NS & 0.96 & $0.40-2.35$ & NS & 1.11 & $0.45-2.70$ & NS \\
\hline Dyslipidemia & 0.38 & $0.19-0.75$ & $<0.01$ & 0.37 & $0.15-0.89$ & 0.03 & 0.47 & $0.20-1.10$ & NS & 0.37 & $0.16-0.85$ & 0.02 \\
\hline Hypertension & 0.88 & $0.44-1.83$ & NS & 0.31 & $0.13-0.73$ & 0.01 & 0.32 & $0.13-0.76$ & 0.01 & 0.34 & $0.14-0.80$ & 0.01 \\
\hline eGFR, $1 \mathrm{~mL} / \mathrm{min} / 1.73 \mathrm{~m}^{2}$ increase & 0.96 & $0.94-0.98$ & $<0.01$ & 0.94 & $0.91-0.97$ & $<0.01$ & 0.94 & $0.91-0.97$ & $<0.01$ & 0.94 & $0.91-0.96$ & $<0.01$ \\
\hline Atrial fibrillation & 1.05 & $0.53-2.07$ & NS & 1.22 & $0.51-2.80$ & NS & 1.07 & $0.45-2.43$ & NS & 1.25 & $0.54-2.80$ & NS \\
\hline Ejection fraction, $1 \%$ increase & 1.00 & $0.97-1.02$ & NS & 0.98 & $0.96-1.01$ & NS & 0.99 & $0.97-1.01$ & NS & 0.99 & $0.97-1.01$ & NS \\
\hline $\mathrm{AA}, 1 \mu \mathrm{g} / \mathrm{mL}$ increase & 0.99 & $0.98-1.00$ & 0.01 & 1.00 & $0.99-1.01$ & NS & & - & & & - & \\
\hline DGLA, $1 \mu \mathrm{g} / \mathrm{mL}$ increase & 0.92 & $0.88-0.96$ & $<0.01$ & & - & & 0.94 & 0.89-0.99 & 0.03 & & - & \\
\hline DGLA/AA, 0.01 increase & 0.92 & $0.85-0.98$ & 0.01 & & - & & & - & & 0.87 & $0.78-0.98$ & 0.02 \\
\hline
\end{tabular}


patients with ACS, none of the PUFA levels were associated with prognosis.

A recent study reported that low levels of omega-6 PUFAs (the sum of AA and DGLA levels) was associated with poor prognosis in patients with ADHF [26]. However, it is well known that AA and DGLA have opposing effects, partly through their metabolites. PUFA metabolites form precursors to prostaglandins, thromboxanes, and leukotrienes [27, 28]. AA is converted to series 2 prostaglandins and series 4 leukotrienes, which have pro-inflammatory potential and the ability to induce platelet aggregation and vasoconstriction $[29,30]$. Although AA is associated with inflammation, atherosclerosis, and a hypercoagulable state, DGLA is converted to series 1 prostaglandins and series 3 leukotrienes, which have anti-inflammatory effects [5, 22, 31-33]. These metabolites also inhibit the effects of AA-derived metabolites. Moreover, a high DGLA/AA ratio indicates a decrease in the endogenous production of AA by delta- 5 desaturase. This may explain the independent association of high DGLA levels and a high DGLA/AA ratio, but not AA levels, with better prognosis in this study population.

Omega-3 PUFA derivatives play a significant role in the process of blood coagulation and inflammation [34, 35]. Many studies have reported that omega-3 PUFAs help lower the risk of cardiovascular diseases. However, in the present study, omega-3 PUFAs were not associated with long-term mortality. Therefore, further studies are warranted to clarify the effects of omega-3 PUFAs, unlike omega-6 PUFAs, in the treatment of acute cardiovascular diseases.

\section{Limitations}

The present study had several limitations. This study was performed in a single institution, and the study population was small. Moreover, the study duration was short; therefore, it may be difficult to generalize the results. Studies with a larger sample size and longer duration are needed to assess the association between PUFAs and prognosis in patients with cardiovascular diseases.

\section{Conclusion}

Low serum DGLA levels and a low DGLA/AA ratio predict long-term mortality in patients with acute cardiovascular diseases, particularly those with ADHF, suggesting that low serum levels of omega-6 PUFAs may be a useful predictor and potential therapeutic target in these patients.

\section{Additional file}

Additional file 1: Serum fatty acids profiles of survivors and

nonsurvivors. (XLSX $32 \mathrm{~kb}$ )

\section{Abbreviations}

AA: Arachidonic acid; ACS: Acute coronary syndrome; ADHF: Acute decompensated heart failure; BMI: Body mass index; Cls: Confidence intervals; DGLA: Dihomo-gamma-linolenic acid; DHA: Docosahexaenoic acid; eGFR: Estimated glomerular filtration rate; EPA: Eicosapentaenoic acid; HDL-C: High-density lipoprotein cholesterol; HRs: Hazard ratios; LDLC: Low-density lipoprotein cholesterol; PUFA: Polyunsaturated fatty acid; TG: Triglyceride

\section{Acknowledgments}

The authors would like to thank Enago (www.enago.jp) for the English language review.

Availability of data and materials

Serum fatty acids profiles of survivors and nonsurvivors are attached as Additional file 1.

Funding

This study was supported partly by JSPS KAKENHI Grant Number JP15K00845.

\section{Authors' contributions}

TM and HD acquired permission from the Ethical Committee. Data sampling was performed by TM and MH with the help of YS, MS, AM, TK, SS, TK, and KM. SO, TA, TS, and ST performed the serum fatty acids analysis. SO, TM, and KS helped in statistical analysis. The manuscript was drafted by SO, TM, and KS. All authors read and approved the final manuscript.

Ethics approval and consent to participate

This study was approved by the Ethical Committee of Juntendo University Hospital. All participants provided written informed consent.

\section{Consent for publication}

Participants were informed of data sharing with their name and identity hidden per consent.

\section{Competing interests}

Dr. Daida has received scholarship funds and lecture fees from Takeda Pharmaceutical Company Ltd. Dr. Miyauchi and Dr. Shimada have also received lecture fees from Mochida Pharmaceutical Company Ltd. and Takeda Pharmaceutical Company Ltd., respectively. The remaining authors report no conflicts of interest.

\section{Publisher's Note}

Springer Nature remains neutral with regard to jurisdictional claims in published maps and institutional affiliations.

Received: 27 March 2017 Accepted: 2 August 2017

Published online: 14 August 2017

\section{References}

1. Webster CM, Deline ML, Watts JL. Stress response pathways protect germ cells from omega-6 polyunsaturated fatty acid-mediated toxicity in Caenorhabditis Elegans. Dev Biol. 2013;373:14-25.

2. Harris WS, Mozaffarian D, Rimm E, Kris-Etherton P, Rudel LL, Appel LJ, Engler MM, Engler MB, Sacks F. Omega-6 fatty acids and risk for cardiovascular disease: a science advisory from the American Heart Association nutrition Subcommittee of the Council on nutrition, physical activity, and metabolism; council on cardiovascular nursing; and council on epidemiology and prevention. Circulation. 2009;119:902-7.

3. Calder PC. Omega-3 polyunsaturated fatty acids and inflammatory processes: nutrition or pharmacology? Br J Clin Pharmacol. 2013;75:645-62.

4. Ghosh S, DeCoffe D, Brown K, Rajendiran E, Estaki M, Dai C, Yip A, Gibson DL. Fish oil attenuates omega-6 polyunsaturated fatty acid-induced dysbiosis and infectious colitis but impairs LPS dephosphorylation activity causing sepsis. PLoS One. 2013;8:e55468

5. Russo GL. Dietary n-6 and n-3 polyunsaturated fatty acids: from biochemistry to clinical implications in cardiovascular prevention. Biochem Pharmacol. 2009;77:937-46.

6. Yanagisawa N, Shimada K, Miyazaki T, Kume A, Kitamura Y, Ichikawa R, Ohmura H, Kiyanagi T, Hiki M, Fukao K, et al. Polyunsaturated fatty acid levels of serum and red blood cells in apparently healthy Japanese subjects living in an urban area. J Atheroscler Thromb. 2010;17:285-94.

7. Bang HO, Dyerberg J, Hjoorne N. The composition of food consumed by Greenland Eskimos. Acta Med Scand. 1976;200:69-73. 
8. Iso H, Kobayashi M, Ishihara J, Sasaki S, Okada K, Kita Y, Kokubo Y, Tsugane S, Group JS. Intake of fish and n3 fatty acids and risk of coronary heart disease among Japanese: the Japan public health center-based (JPHC) study cohort I. Circulation. 2006:113:195-202.

9. Yokoyama M, Origasa H, Matsuzaki M, Matsuzawa Y, Saito Y, Ishikawa Y, Oikawa S, Sasaki J, Hishida H, Itakura H, et al. Effects of eicosapentaenoic acid on major coronary events in hypercholesterolaemic patients (JELIS): a randomised open-label, blinded endpoint analysis. Lancet. 2007;369:1090-8.

10. GISSI-Prevenzione Investigators. Dietary supplementation with n-3 polyunsaturated fatty acids and vitamin $\mathrm{E}$ after myocardial infarction: results of the GISSI-Prevenzione trial. Lancet. 1999;354:447-55.

11. Marchioli R. Early protection against sudden death by n-3 polyunsaturated fatty acids after myocardial infarction: time-course analysis of the results of the gruppo italiano per lo studio della sopravvivenza nell'infarto miocardico (gissi)-prevenzione. Circulation. 2002;105:1897-903.

12. Marchioli R, Levantesi G, Macchia A, Maggioni AP, Marfisi RM, Silletta MG, Tavazzi L, Tognoni G, Valagussa F, Investigators GI-P. Antiarrhythmic mechanisms of n-3 PUFA and the results of the GISSI-Prevenzione trial. J Membr Biol. 2005:206:117-28

13. Kristensen $S$, Schmidt EB, Schlemmer A, Rasmussen C, Lindgreen E, Johansen MB, Christensen $\mathrm{JH}$. The effect of marine n-3 polyunsaturated fatty acids on cardiac autonomic and hemodynamic function in patients with psoriatic arthritis: a randomised, double-blind, placebocontrolled trial. Lipids Health Dis. 2016;15:216.

14. Yang ZH, Inoue S, Taniguchi $Y$, Miyahara H, Iwasaki Y, Takeo J, Sakaue H, Nakaya Y. Long-term dietary supplementation with saury oil attenuates metabolic abnormalities in mice fed a high-fat diet: combined beneficial effect of omega-3 fatty acids and long-chain monounsaturated fatty acids. Lipids Health Dis. 2015;14:155.

15. Nodari S, Triggiani M, Campia U, Manerba A, Milesi G, Cesana BM, Gheorghiade M, Dei Cas L. Effects of $n-3$ polyunsaturated fatty acids on left ventricular function and functional capacity in patients with dilated cardiomyopathy. J Am Coll Cardiol. 2011;57:870-9.

16. Gissi-Hf investigators. Effect of $n-3$ polyunsaturated fatty acids in patients with chronic heart failure (the GISSI-HF trial): a randomised, double-blind, placebo-controlled trial. Lancet. 2008:372:1223-30.

17. Ghio S, Scelsi L, Latini R, Masson S, Eleuteri E, Palvarini M, Vriz O, Pasotti M, Gorini M, Marchioli R, et al. Effects of $n-3$ polyunsaturated fatty acids and of rosuvastatin on left ventricular function in chronic heart failure: a substudy of GISSI-HF trial. Eur J Heart Fail. 2010;12:1345-53.

18. Katan MB. Omega-6 polyunsaturated fatty acids and coronary heart disease. Am J Clin Nutr. 2009;89:1283-4.

19. David E, Laaksonen M, Nyyssönen $K$, Niskanen L, Rissanen TH, Jukka T, Salonen M. Prediction of cardiovascular mortality in middle-aged men by dietary and serum Linoleic and polyunsaturated fatty acids. Arch Intern Med. 2005;165:193-9.

20. Farvid MS, Ding M, Pan A, Sun Q, Chiuve SE, Steffen LM, Willett WC, Hu FB. Dietary linoleic acid and risk of coronary heart disease: a systematic review and meta-analysis of prospective cohort studies. Circulation. 2014;130:1568-78.

21. Petrone AB, Weir N, Hanson NQ, Glynn R, Tsai MY, Gaziano JM, Djousse L. Omega-6 fatty acids and risk of heart failure in the Physicians' health study. Am J Clin Nutr. 2013;97:66-71.

22. Al-Khudairy L, Hartley L, Clar C, Flowers N, Hooper L, Rees K. Omega 6 fatty acids for the primary prevention of cardiovascular disease. Cochrane Database Syst Rev. 2015;11:CD011094.

23. McKee PA, Castelli WP, McNamara PM, Kannel WB. The natural history of congestive heart failure: the Framingham study. N Engl J Med. 1971;285:1441-6.

24. Shimamoto K, Ando K, Fujita T, Hasebe N, Higaki J, Horiuchi M. The Japanese Society of Hypertension Guidelines for the Management of Hypertension (JSH 2014). Hypertens Res. 2014;37:253-3.

25. Matsuo S, Imai E, Horio M, Yasuda Y, Tomita K, Nitta K, Yamagata K, Tomino Y, Yokoyama H, Hishida A. Revised equations for estimated GFR from serum creatinine in Japan. Am J Kidney Dis. 2009;53:982-92.

26. Nagai T, Honda Y, Sugano Y, Nishimura K, Nakai M, Honda S, Iwakami N, Okada A, Asaumi Y, Aiba T, et al. Circulating omega-6, but not omega-3 polyunsaturated fatty acids, are associated with clinical outcomes in patients with acute decompensated heart failure. PLoS One. 2016;11:e0165841.

27. Das UN. Essential fatty acids - a review. Curr Pharm Biotechnol. 2006;7:467-82.

28. Lin N, Shi JJ, Li YM, Zhang XY, Chen Y, Calder PC, Tang LJ. What is the impact of $n-3$ PUFAs on inflammation markers in type 2 diabetic mellitus populations?: a systematic review and meta-analysis of randomized controlled trials. Lipids Health Dis. 2016;15:133.

29. Wiktorowska-Owczarek A, Berezińska M, Nowak J. PUFAs: structures, metabolism and functions. Adv Clin Exp Med. 2015:24:931-41.

30. Marion-Letellier R, Savoye G, Ghosh S. Polyunsaturated fatty acids and inflammation. IUBMB Life. 2015;67:659-67.

31. Wu JH, Lemaitre RN, King IB, Song X, Psaty BM, Siscovick DS, Mozaffarian D. Circulating omega- 6 polyunsaturated fatty acids and total and cause-specific mortality: the cardiovascular health study. Circulation. 2014;130:1245-53.

32. Kawashima H, Toyoda-Ono Y, Suwa Y, Kiso Y. Subchronic (13-week) oral toxicity study of dihomo-gamma-linolenic acid (DGLA) oil in rats. Food Chem Toxicol. 2009;47:1280-6.

33. Kapoor R, Huang YS. Gamma linolenic acid: an antiinflammatory omega-6 fatty acid. Curr Pharm Biotechnol. 2006;7:531-4.

34. Oh DY, Talukdar S, Bae EJ, Imamura T, Morinaga H, Fan W, Li P, Lu WJ, Watkins SM, Olefsky JM. GPR120 is an omega-3 fatty acid receptor mediating potent anti-inflammatory and insulin-sensitizing effects. Cell. 2010;142:687-98.

35. Mishra A, Chaudhary A, Sethi S. Oxidized omega-3 fatty acids inhibit NF-kappaB activation via a PPARalpha-dependent pathway. Arterioscler Thromb Vasc Biol. 2004;24:1621-7.

\section{Submit your next manuscript to BioMed Central and we will help you at every step:}

- We accept pre-submission inquiries

- Our selector tool helps you to find the most relevant journal

- We provide round the clock customer support

- Convenient online submission

- Thorough peer review

- Inclusion in PubMed and all major indexing services

- Maximum visibility for your research

Submit your manuscript at www.biomedcentral.com/submit
Biomed Central 